\title{
Hemophagocytic Syndrome Associated with Scrub Typhus: A Case Report from North East India
}

\section{Tony Ete ${ }^{1 *}$, Akash Roy ${ }^{1}$, Bhupen Barman ${ }^{2}$, Kyrshanlang G Lynrah², Ojing Komut ${ }^{3}$ and Yookarin Khonglah ${ }^{4}$}

${ }^{1}$ Department of General Medicine, North Eastern Indira Gandhi Regional Institute of Health and Medical Sciences Shillong, Meghalaya, India

${ }^{2}$ Department of Medicine, North Eastern Indira Gandhi Regional Institute of Health and Medical Sciences, Shillong, Meghalaya, India

${ }^{3}$ Department of General Surgery, North Eastern Indira Gandhi Regional Institute of Health and Medical Sciences, Shillong, Meghalaya, India

${ }^{4}$ Department of Pathology, North Eastern Indira Gandhi Regional Institute of Health and Medical Sciences, Shillong, Meghalaya, India

\begin{abstract}
Scrub typhus infection is an important aetiology of acute undifferentiated fever in south-east Asia and India. Haemophagocytic Lymphohistiocytosis (HLH) (haemophagocytic syndrome) is a potentially fatal hyper inflammatory syndrome that is characterized by histiocyte proliferation and haemophagocytosis. We describe a case of Haemphagocytic syndrome secondary to scrub typhus which presented with fever, rash, pancytopenia, epistaxis and haematuria who responded dramatically with respect to haematological parameters and clinically following prompt antimicrobial therapy. Scrub typhus with hemophagocytic syndrome can be complicated by multiorgan failure. Patients with scrub typhus usually have an excellent response to treatment; therefore, early diagnosis and prompt administration of antimicrobial therapy may prevent the development of serious complications.
\end{abstract}

Keywords: Scrub typhus; Eschar; Pancytopenia; Hemophagocytic syndrome

\section{Introduction}

Scrub typhus infection is an important aetiology of acute undifferentiated fever in south-east Asia and India [1,2]. It is a zoonotic rickettsial illness caused by Orientia tsutsugamushi and is endemic in the "Tsutsugamushi triangle" that extends from northern Japan and far eastern Russia to northern Australia in the south and Pakistan in the west [3]. The reservoirs for infection are the chiggers (larva of trombiculid mite), rats and humans are accidentally infected. It is transmitted by trombiculid mites in long grasses and in dirtfloor homes, with infection characterized by a flu-like illness of fever, headache and myalgia lasting approximately one week. In some, the illness progresses to multiple organ dysfunction syndrome and death.

Haemophagocytic lymphohistiocytosis (HLH), also known as Hemophagocytic Syndrome (HPS) is a fatal hyperinflammatory syndrome that may be inherited or secondary to any severe infection, malignancy or rheumatological condition and occurring at any age. The diagnosis is established by fulfilling one of the following criteria [4] (i) A molecular diagnosis consistent with haemophagocytic syndrome (e.g. PRF mutations, SAP mutations, MUNC13-4 mutations) (ii) having five out of eight of the following: fever, splenomegaly, cytopenia (affecting more than two cell lineages), hypertriglyceridaemia and/or hypofibrinogenaemia, haemophagocytosis in the bone marrow, spleen, or lymph nodes without evidence of malignancy; low or absent Natural Killer (NK) cell cytotoxicity, hyperferritinaemia and elevated soluble CD25.

We hereby describe a case of a 38 year old male with the development of Haemophagocytic Lymphohistiocytosis (HLH) secondary to rickettsial infection of Orientia tsutsugamushi which is a rare presentation of the syndrome.

\section{Case Report}

38 years old male presented with fever for two weeks preceded by history of travel in densely forested areas. Fever was high grade $(1020 \mathrm{~F})$, hectic and continuous in nature. There was no associated cough. After one week of fever, the patient developed petechiae and ecchymosis involving the trunk and neck (Figure 1). Patient developed hematuria after seven days of fever. Subsequently he had epistaxis following which he was brought to emergency department. There was

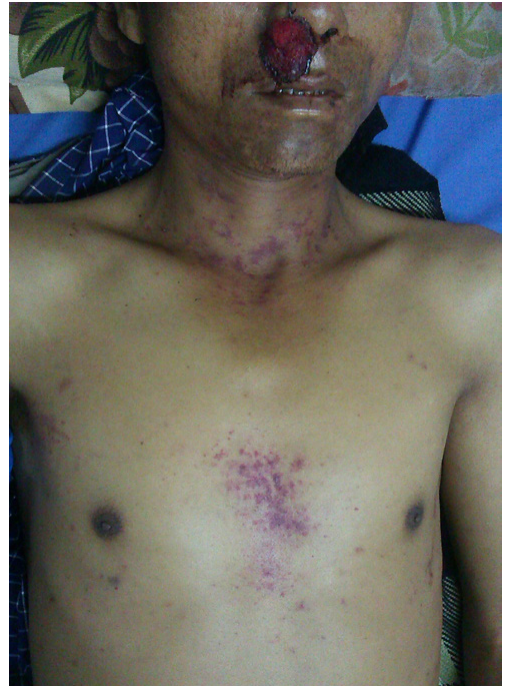

Figure 1: Figure showing petechial and ecchymotic lesions over anterior chest wall and nasal packing given for epistaxis.

no history of intake of any drug. Patient had no past history of any major illness. On examination, the patient was alert, conscious and cooperative. Pulse was 110/minute, BP-100/60 mmHg; respiratory rate was $24 /$ minute. Oxygen saturation was low (86\%). Abdomen was soft and non-tender with no organomegaly. Petechiae were present in the intramammary region and right anterior axillary region. No lymph nodes were palpable. An eschar was present on the medial aspect of

*Corresponding author: Tony Ete, Department of General Medicine, North Eastern Indira Gandhi Regional Institute of Health and Medical Sciences Shillong, Meghalaya, India, Tel: 0364-2538013; E-mail: tonyete14@gmail.com

Received August 24, 2015; Accepted October 04, 2015; Published October 11 2015

Citation: Ete T, Roy A, Barman B, Lynrah KG, Komut O, et al. (2015) Hemophagocytic Syndrome Associated with Scrub Typhus: A Case Report from North East India. J Clin Case Rep 5: 606. doi:10.4172/2165-7920.1000606

Copyright: @ 2015 Ete T, et al. This is an open-access article distributed under the terms of the Creative Commons Attribution License, which permits unrestricted use, distribution, and reproduction in any medium, provided the original author and source are credited. 
right thigh (Figure 2). Blood report revealed Hb-3.8 gm\%, TLC- 500/ $\mathrm{mm}^{3}$, N14L68M18E1, platelets- $<10,000 / \mathrm{mm}^{3}$, ESR- $08 \mathrm{~mm}$, PCV$11.5 \%, \mathrm{MCV}-113 \mathrm{fl}, \mathrm{MCH}-37 \mathrm{pg}$, MCHC-33\%. Peripheral blood smear showed anisocytosis, microcytic and hypochromic anaemia with macrocytes and target cells. Leucopenia with few lymphocytes and monocytes and also including atypical cells. Liver function test revealed SGOT-104 U/L, SGPT-151 U/L, ALP-341 U/L, albumin - $2.5 \mathrm{gm} / \mathrm{dl}$, globulin-2 gm/dl. Blood for HIV-1, HIV-2, Hepatitis B Surface Antigen and anti HCV were negative. Blood glucose was $131 \mathrm{mg} / \mathrm{dl}$, urea-82 mg/ $\mathrm{dl}$, and creatinine- $1.7 \mathrm{mg} / \mathrm{dl}$. Widal test was negative. Serum Ferritin was $1400 \mathrm{ng} / \mathrm{ml}$. Chest X-ray revealed bilateral non homogenous opacities. Sonography of abdomen revealed no abnormality. Blood for malaria dual antigen was negative. Blood for NS1 antigen, dengue IgM and leptospira IgM were negative. Blood for scrub IgM was sent which came out to be positive done through immunochromatographic assay. Initially the patient was shifted to ICU, was put on oxygen inhalation, and treated with intravenous fluids, antibiotics (intravenous ceftriaxone and doxycycline) and platelet transfusion so as to decrease the risk of spontaneous bleeding. Bone marrow aspiration (BMA) was done to rule out underlying hematological malignancy. However, Bone marrow features showed phagocytic histiocytes ingesting self hematopoietic cells suggestive of HPS (Figure 3). The patient was started on doxycycline.

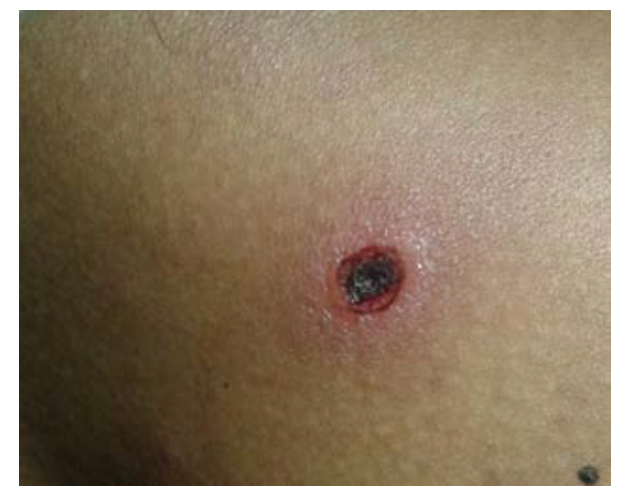

Figure 2: Eschar of scrub typhus.

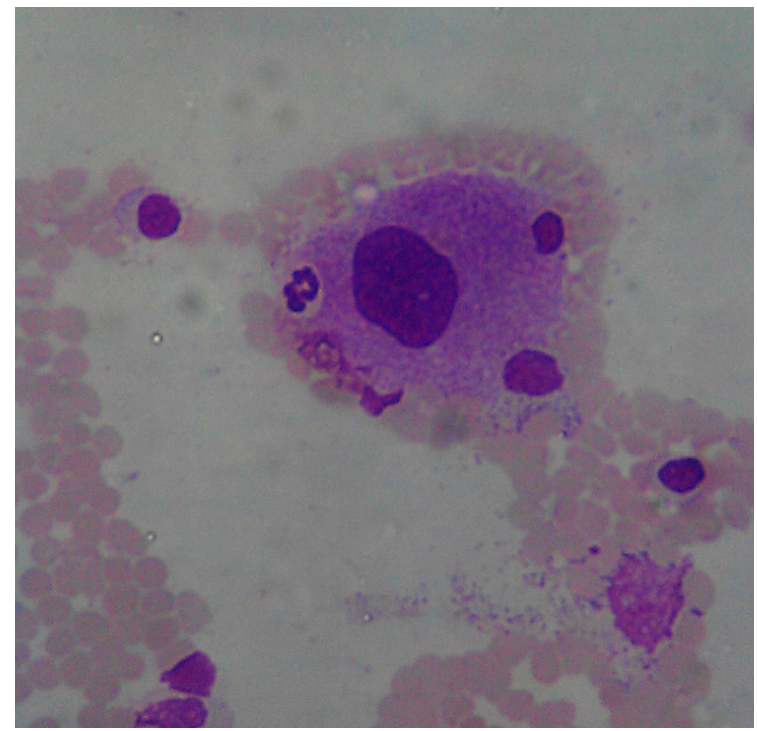

Figure 3: Bone marrow aspiration showing phagocytic histiocytes ingesting self hematopoietic cells.
On the third day of treatment azithromycin was also added. The patient showed features of clinical improvement on seventh day of treatment. On the tenth day of treatment the patient's blood parameters were $\mathrm{Hb}$ 9.6 gm\%, TLC-7,500/cumm, N50L36M14E0, platelets-1.8 lakhs/cumm, ESR-9 mm/hr, PCV-28.9\%, MCV-98 fl, MCH-52 pg, MCHC-33\%. The patient was discharged after more than two weeks in hospital in stable condition with an advice to attend outpatient department in follow up.

\section{Discussion}

Hemophagocytosis Syndrome (HPS) or Hemophagocytic Lymphohistiocytosis (HLH) is a rare hematological disorder characterized by febrile illness, cytopenia, lymphadenopathy and hepatosplenomegaly and hyperferritinemia [5]. Phagocytosis of blood cells and their precursors is a hallmark of hemophagocytic syndromes (HPS). Hemophagocytosis is achieved mostly by monocytes and macrophages. Nitroblue tetrazolium reduction by monocytes from patients with HPS is approximately six times that of control monocytes. Excessive activation of monocytes in HLH may be due to stimulation by high levels of activating cytokines. High levels of interferon- $\gamma$ (IFN $\gamma$ ), soluble interleukin-2 receptor, Tumor Necrosis Factor-A (TNFa), interleukin-1, and interleukin- 6 have been demonstrated to be involved in HPS, suggesting the elaboration of activating cytokines by T-helper cells. It can also result from poorly regulated or inappropriate Th-1 responses to intracellular pathogens. The details of immunological protective mechanisms against rickettsial infections, including activation of cytokines, are not fully understood, but macrophages and T-cells are believed to play an important role in protection against rickettsial infections and appearance of HPS. HPS is caused by a number of viruses, including Cytomegalovirus (CMV), Epstein Barr Virus (EBV), human herpes virus-6 as well as malignancies like T-cell lymphomas and collagen vascular diseases [6]. Hemophagocytosis causing pancytopenia is a medical emergency and may be fatal despite aggressive treatment.

Scrub typhus is usually endemic in South East Asia, northern Australia and pacific islands [7]. In India it has been reported from Himalayan regions, Haryana and southern India. Furthermore, Scrub typhus has been frequently been reported from North East India and associated with major clinical complications [8]. Scrub typhus is an acute febrile illness which results from the bite of infected larval form of mite, called chigger, in endemic areas. Following, an incubation period of 7 to 10 days, the nonspecific prodrome of pyrexia, skin rash, myalgia, gastrointestinal disturbances, and lymphadenopathy starts. Although not consistently seen, the most pathognomonic sign of scrub typhus is an eschar that develops at the site of mite bite. During human infection, O. tsutsugamushi, being a rickettsial organism, selectively targets the vascular endothelial cells of the small to medium-sized blood vessels. However, it can also invade underlying tissues such as smooth muscle cells, perivascular macrophages, and monocytes. Consequently, widespread vasculitis or perivasculitis is the hallmark pathophysiologic mechanism implicated in Multiorgan Dysfunction Syndrome (MODS) in patients with severe infection [9]. Our patient was diagnosed to have scrub typhus by the positivity in the immunochromatographic card test for detection of IgM antibodies to Orientia tsutsugamushi. The clinical profile of our patient along with serological evidence of scrub typhus and evidence of haemophagocytosis enabled us to make the diagnosis of haemophagocytosis associated with scrub typhus infection. Furthermore, the response to anti R. tsutsugamushi medications further corroborated our diagnosis. Pancytopenia associated with scrub typhus has been rarely reported in the medical literature [10]. Mortality rates of untreated patients have ranged from $0-30 \%$. The 
Citation: Ete T, Roy A, Barman B, Lynrah KG, Komut O, et al. (2015) Hemophagocytic Syndrome Associated with Scrub Typhus: A Case Report from North East India. J Clin Case Rep 5: 606. doi:10.4172/2165-7920.1000606

prognosis of scrub typhus associated with HPS is unknown [11]. Inflammatory responses of humans appear to coincide with the disease severity in scrub typhus, and the cytotoxic T-cell mediated macrophage over activity may induce hemophagocytosis in susceptible individuals [12]. This hypothesis has been further substantiated by the observation of increased serum levels of IFN- $\gamma$, M-CSF and TNF- $\alpha$ in patient with scrub typhus in several studies [13]. In a review by Basheer et al. [9] 21 cases of haemophagocytic syndrome associated with scrub typhus was described out of which 14 were of mild to moderate nature.

\section{Conclusion}

HPS though rare, should be considered in severe cases of scrub typhus especially if associated with cytopenias, liver dysfunction, and coagulation abnormalities especially in endemic areas like India. Furthermore, wherever possible, a diagnosis of primary HPS should always be excluded in these cases by appropriate mutation analysis studies. However, it is always beneficial to start antirickettsial chemotherapy empirically in these patients in places where diagnostic facilities are not readily available.

\section{References}

1. Silpapojakul K (1997) Scrub typhus in the Western Pacific region. Ann Acad Med Singapore 26: 794-800.

2. Chrispal A, Boorugu H, Gopinath KG, Chandy S, Prakash JA, et al. (2010) Acute undifferentiated febrile illness in adult hospitalized patients: the disease spectrum and diagnostic predictors - an experience from a tertiary care hospital in South India. Trop Doct 40: 230-234

3. Sharma P, Kakkar R, Kaore SN, Vadav VK, Sharma R (2010) Geographical distribution, effect of season and life cycle of scrub typhus. JK Science 12: 63-64.
4. Henter JI, Horne A, Arico M, Egeler RM, Filipovich A H, et al.( 2007) HLH-2004 diagnostic and therapeutic guidelines for hemophagocytic lymphohistiocytosis. Pediatr Blood Cancer 48: 124-131.

5. Rouphael NG, Talati NJ, Vaughan C, Cunningham K, Moreira R, et al. (2007) Infections associated with haemophagocytic syndrome. Lancet Infect Dis 7: 81422.

6. Iwasaki H, Hashimoto K, Takada N, Nakayama T, Ueda T, et al. (1994) Fulminant Rickettsia tsutsugamushi infection associated with haemophagocytic syndrome. Lancet 343: 1236

7. Mathai E, Lloyd G, Cherian T, Abraham OC, Cherian AM (2001) Serological evidence for the continued presence of human rickettsioses in southern India. Ann Trop Med Parasitol 95: 3958.

8. Jamil M (2014) "Clinical Manifestations and Complications of Scrub Typhus: A Hospital Based Study from North Eastern India." Journal of the Association of Physicians of India 62: 19.

9. Basheer A, Padhi S, Boopathy V, Mallick S, Nair S, et al. (2015) Hemophagocytic Lymphohistiocytosis: an Unusual Complication of Orientia tsutsugamushi Disease (Scrub Typhus). Mediterr J Hematol Infect Dis 7: e2015008

10. Premaratna R, Williams HS, Chandrasena TG, Rajapakse RP, Kularatna SA, et al. (2009) Unusual pancytopenia secondary to haemophagocytosis syndrome in rickettsioses. Trans R Soc Trop Med Hyg 103: 9613.

11. Chaudhry D, Garg A, Singh I, Tandon C, Saini R (2009) Rickettsial diseases in Haryana: not an uncommon entity. J Assoc Physicians India 57: 3347.

12. Sahni SK, Rydkina E (2009) Host cell interactions with pathogenic Rickettsia species. Future Microbiol 4: 323-339.

13. Iwasaki H, Takada N, Nakamura T, Ueda T (1997) Increased levels of macrophage colony stimulating factor, gamma interferon and tumour necrosis factor alpha in sera of patients with Orientia tsutsugamushi infection. $\mathrm{J}$ Clin Microbiol 35: 3320-3322. 